\title{
Identification and assessment of markers of biotin status in healthy adults
}

\author{
Wei Kay Eng ${ }^{1}$, David Giraud ${ }^{1}$, Vicki L. Schlegel ${ }^{2}$, Dong Wang ${ }^{3}$, Bo Hyun Lee ${ }^{2}$ and Janos Zempleni ${ }^{1 *}$ \\ ${ }^{1}$ Departments of Nutrition and Health Sciences, University of Nebraska-Lincoln, Lincoln, NE 68583-0806, USA \\ ${ }^{2}$ Food Science and Technology, University of Nebraska-Lincoln, Lincoln, NE 68583-0919, USA \\ ${ }^{3}$ Statistics, University of Nebraska-Lincoln, Lincoln, NE 68583-0963, USA \\ (Submitted 13 July 2012 - Final revision received 16 October 2012 - Accepted 16 October 2012 - First published online 10 January 2013)
}

\section{Abstract}

Human biotin requirements are unknown and the identification of reliable markers of biotin status is necessary to fill this knowledge gap. Here, we used an outpatient feeding protocol to create states of biotin deficiency, sufficiency and supplementation in sixteen healthy men and women. A total of twenty possible markers of biotin status were assessed, including the abundance of biotinylated carboxylases in lymphocytes, the expression of genes from biotin metabolism and the urinary excretion of biotin and organic acids. Only the abundance of biotinylated 3-methylcrotonyl-CoA carboxylase (holo-MCC) and propionyl-CoA carboxylase (holo-PCC) allowed for distinguishing biotin-deficient and biotin-sufficient individuals. The urinary excretion of biotin reliably identified biotin-supplemented subjects, but did not distinguish between biotin-depleted and biotin-sufficient individuals. The urinary excretion of 3-hydroxyisovaleric acid detected some biotin-deficient subjects, but produced a meaningful number of false-negative results and did not distinguish between biotinsufficient and biotin-supplemented individuals. None of the other organic acids that were tested were useful markers of biotin status. Likewise, the abundance of mRNA coding for biotin transporters, holocarboxylase synthetase and biotin-dependent carboxylases in lymphocytes were not different among the treatment groups. Generally, datasets were characterised by variations that exceeded those seen in studies in cell cultures. We conclude that holo-MCC and holo-PCC are the most reliable, single markers of biotin status tested in the present study.

Key words: Biotin status assessment: Carboxylases: Gene expression: Organic acids

Biotin is a water-soluble vitamin that serves as a coenzyme for five human carboxylases, namely acetyl-CoA carboxylases (ACC) 1 and 2, pyruvate carboxylase, propionyl-CoA carboxylase (PCC) and 3-methylcrotonyl-CoA carboxylase (MCC) ${ }^{(1)}$. These enzymes play an important role in the metabolism of fatty acids, glucose and amino acids. Histones $\mathrm{H} 3$ and $\mathrm{H} 4$ are also modified by covalent attachment of biotin ${ }^{(2-5)}$. Biotinylation of histones is a rare event, and less than $0.001 \%$ of histones $\mathrm{H} 3$ and $\mathrm{H} 4$ are biotinylated ${ }^{(6-8)}$. Biotinylated histones are over-represented in repressed $\operatorname{loci}^{(4,5,9)}$ and, despite their rarity, biotinylation marks contribute to chromatin condensation $^{(10)}$. The binding of biotin to both carboxylases and histones is catalysed by holocarboxylase synthetase ${ }^{(1,11)}$.

Despite the essential roles of biotin in intermediary metabolism and gene regulation, human biotin requirements are unknown. Consequently, only recommendations for adequate intake, but no RDA, are available for biotin ${ }^{(12)}$. Recommendations for adequate intake are based solely on biotin intake in the general, apparently healthy, population ${ }^{(12)}$. This approach is flawed in the case of biotin, where dietary intake data are only crude estimates. Currently, no studies are available quantifying biotin in foods using chemically specific assays ${ }^{(13)}$, and it is unclear whether intake estimates exceed or underestimate the true biotin intake. Also, the 'normal state' is defined using biotin-dependent enzymes or urinary biotin metabolites as markers, while ignoring the changes occurring at the chromatin level.

As of today, no comprehensive study has been published that investigated markers of human biotin status from various possible angles, including carboxylase biotinylation, metabolome and transcriptome in healthy adults. The identification of sensitive and robust markers of biotin status would represent a major advance in our pursuit of the quantification of human biotin requirements. In the present study, we devised a biotin feeding protocol to create states of biotin depletion, biotin sufficiency and biotin supplementation in healthy women and men. We assessed the suitability of twenty distinct markers to identify those that reliably discriminate among the subjects in the three treatment groups. Only two markers met our stringent requirements and can be

Abbreviations: 3-HIA, 3-hydroxyisovaleric acid; ACC, acetyl-CoA carboxylase; HABA, 2-(4'-hydroxyazobenzene)-benzoic acid; MCC, 3-methylcrotonyl-CoA carboxylase; PCC, propionyl-CoA carboxylase.

*Corresponding author: J. Zempleni, fax +1 402472 1587, email jzempleni2@unl.edu 


\section{Subjects and methods}

\section{Study principle}

The present study is a randomised cross-over outpatient study. Defined states of biotin supply were established by supplementing the study subjects with drinks containing spraydried egg-white. Egg-white contains the protein avidin, which binds biotin tightly and makes the vitamin unavailable for intestinal absorption ${ }^{(14,15)}$. Graded levels of chemically pure biotin were added back to the egg-white shakes, thereby inducing states of biotin depletion, biotin sufficiency and biotin supplementation (given later).

\section{Study subjects}

A total of seventeen apparently healthy adults (seven men and ten women) aged 21-45 years completed the present study. Exclusion criteria included a recent history of smoking ${ }^{(16)}$, inborn errors of biotin metabolism (carboxylase deficiency, holocarboxylase synthetase deficiency and biotinidase deficiency ${ }^{(17,18)}$, use of vitamin supplements, use of anticon-


were tested for pregnancy prior to each period of egg-white feeding by using a commercial pregnancy test. Subjects were allowed to engage in normal daily activities. After the end of the study, one male subject (21 years old) admitted that he did not comply with the study protocol and his data were excluded from the analysis. During the biotin-deficient phase, none of the subjects had frank signs of severe biotin deficiency such as dermatitis, rash and alopecia. The present study was conducted according to the guidelines laid down in the Declaration of Helsinki, and all procedures involving human subjects were approved by the Institutional Review Board of the University of Nebraska-Lincoln, USA. Subjects signed an informed consent prior to enrolling in the study.

\section{Study protocol}

The entire study took each subject 15 weeks to complete (Fig. 1). During the first 2 weeks, subjects consumed a regular, self-selected diet without the use of any vitamin supplements. The objective of this adjustment period was to eliminate excess vitamins from inadvertent use of vitamin supplements before the study began. Subjects were instructed to avoid biotin-rich foods such as yeast, livers, meats, cereals,

mayonnaise and eggs ${ }^{(24)}$, and were provided with a list of foods to avoid. After completion of the adjustment period, each subject completed the following three treatment phases (3 weeks each) ${ }^{(25)}$ in randomised order: biotin depletion (zero dietary biotin, representing levels seen in individuals consuming diets containing raw egg-white and individuals with undiagnosed deficiencies of biotinidase $)^{(26,27)}$, biotin sufficiency $(30 \mu \mathrm{g} / \mathrm{d}$, representing recommendations for adequate intake of biotin $)^{(12)}$ and biotin supplementation $(600 \mu \mathrm{g} / \mathrm{d}$, representing the level of biotin in typical over-the-counter supplement users) ${ }^{(28,29)}$. In the second week of each treatment, participants filled out 3-d dietary records in order to estimate biotin intake. Periods of biotin-defined diets were interrupted by 2 weeks with no biotin treatment. These periods are sufficiently long for biotin levels to return to baseline level ${ }^{(15,25,30)}$. Subjects consumed a regular, self-selected diet (given earlier), modified only by the amounts of biotin during treatment phases.

During treatment, dietary biotin was adjusted by consuming an egg-white drink $(200 \mathrm{ml})$ with every major meal (breakfast, lunch and dinner) of the day. The amount of egg-white in the drinks was chosen as follows. First, the amount of avidin in
(A)

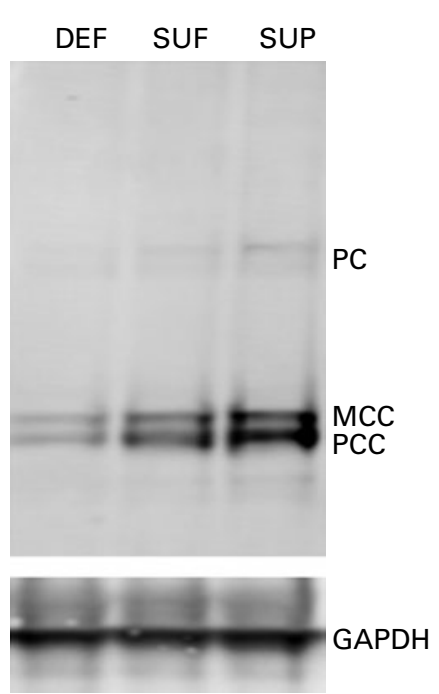

(B)

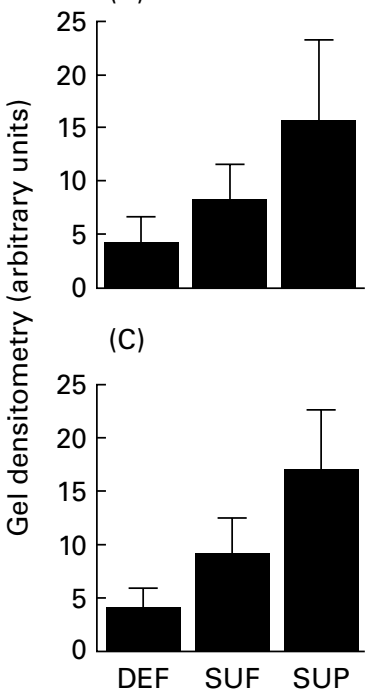

Fig. 2. (A) Abundance of biotinylated (holo-)pyruvate carboxylase (PC), 3-methylcrotonyl-CoA carboxylase (MCC) and propionyl-CoA carboxylase (PCC) in lymphocytes from biotin-deficient (DEF), biotin-sufficient (SUF) and biotin-supplemented (SUP) healthy adults (top gel). Glyceraldehyde-3phosphate dehydrogenase (GAPDH) was used as the loading control (bottom gel). The image was cropped from an original full-size scanned picture. (B) Gel densitometry analysis of holo-MCC $(n 16,0.0003<P<0.0012$ with Bonferroni correction for all possible comparisons). (C) Gel densitometry analysis of holoPCC ( $n$ 16, $P<0.0003$ with Bonferroni correction for all possible comparisons). Values are means, with standard deviations represented by vertical bars. 
(A)

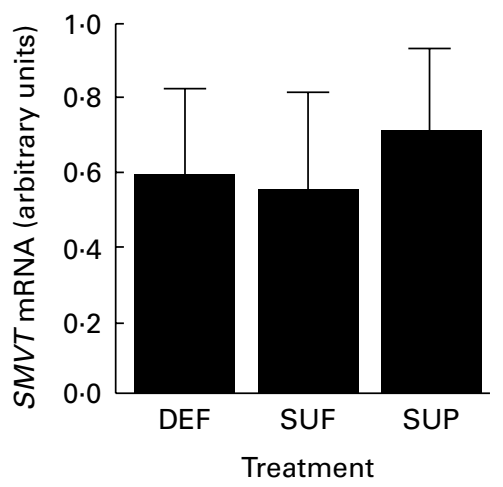

(C)

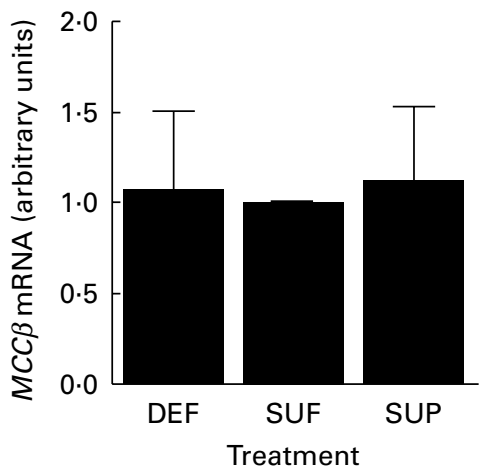

(B)

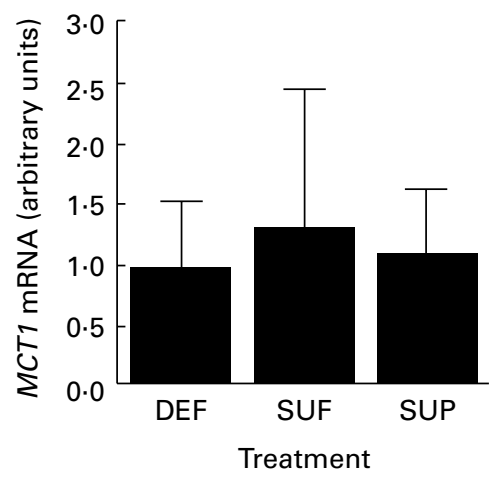

(E)

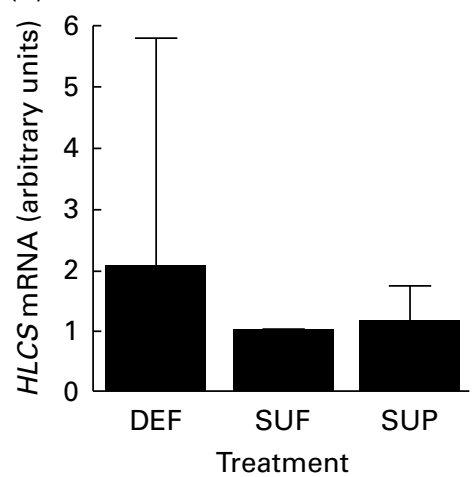

Fig. 3. Abundance of mRNA coding for (A) sodium-dependent multivitamin transporter (SMVT), (B) monocarboxylate transporter 1 (MCT1), (C) 3-methylcrotonylCoA carboxylase $\beta(M C C \beta)$, (D) acetyl-CoA carboxylase 1 (ACC1) and (E) holocarboxylase synthetase (HLCS) in lymphocytes from biotin-deficient (DEF), biotin-sufficient (SUF) and biotin-supplemented (SUP) healthy adults. Values are means, with standard deviations represented by vertical bars (n 16, $0.0784<P<0.9823$ ).

spray-dried egg-white (Barry Farm, Cridersville, OH, USA) was titrated based on the 2-(4'-hydroxyazobenzene)-benzoic acid (HABA) assay $(n 8)^{(31,32)}$. Avidin activity $(\mu \mathrm{g} / \mathrm{ml}$ dried eggwhite) was calculated based on the method of Green ${ }^{(31,32)}$ using the following equation:

$$
\mathrm{AA}=(3 \cdot 1 \times 68000 \times \mathrm{OD} \times 1000) /(3 \times 34000 \times 4),
$$

where AA is avidin activity ( $\mu \mathrm{g} / \mathrm{ml}$ dried egg-white), $3 \cdot 1$ is the sample volume $(\mathrm{ml})$ after the addition of HABA, 68000 molecular weight of avidin, OD is optical density of the supernatant of the egg-white solution plus HABA at $500 \mathrm{~nm}$ corrected by the optical density before HABA, 3 is the sample volume $(\mathrm{ml})$ before the addition of HABA and 4 is the number of avidin molecules in the avidin tetramer.

A measure of $1 \mathrm{~g}$ of egg-white contained approximately $600 \mu \mathrm{g}$ of avidin. Second, we calculated the amount of egg-white needed to bind the biotin in an average diet $(30-70 \mu \mathrm{g} / \mathrm{d})^{(12)}$, including an allowance for microbial biotin synthesis and diets that were inadvertently high in biotin. Assuming that $1 \mu \mathrm{g}$ of avidin binds $0.014 \mu \mathrm{g}$ biotin $^{(32)}$, we prepared egg-white drinks containing $7 \cdot 1 \mathrm{mg}$ of avidin in $200 \mathrm{ml}$ (one serving), which is sufficient to bind about $100 \mu \mathrm{g}$ of biotin. Spray-dried egg-white was dissolved in orange juice to improve palatability; the juices were stored at $4^{\circ} \mathrm{C}$ for up to $2 \mathrm{~d}$ before consumption. This drink was used for depleting subjects of biotin. For biotin-sufficient subjects, biotin was added back to the drinks to saturate all the avidin plus provide an additional $30 \mu \mathrm{g} / \mathrm{d}$. For biotin-supplemented subjects, the amount of available biotin was adjusted to $600 \mu \mathrm{g} / \mathrm{d}$. Our estimates were confirmed by quantifying the content of free biotin in egg-white drinks by using the avidin-binding assay ${ }^{(29)}$

\section{Sample collection}

Blood samples were collected after an overnight fast by an experienced phlebotomist at the University Health Center of Nebraska-Lincoln. A volume of $180 \mathrm{ml}$ of fasting blood was collected after the initial 2-week adjustment period and on the last day of each treatment period. In addition, $30 \mathrm{ml}$ of fasting blood were collected after the 2-week washout period of each treatment. Lymphocytes were isolated by density gradient centrifugation and counted using a haemocytometer ${ }^{(33)}$. Aliquots of twenty million and two million cells were frozen $\left(-80^{\circ} \mathrm{C}\right)$ for subsequent carboxylase analysis and for isolation of total RNA, respectively. Spot urine samples were collected after an overnight fast at the end of each washout and treatment phase. Urine samples were sub-aliquoted to avoid repeated freeze-thaw cycles and frozen at $-20^{\circ} \mathrm{C}$ for the subsequent analyses of organic acids, biotin and creatinine.

\section{Sample analysis}

The abundance of biotinylated (holo-)carboxylases was quantified in lymphocyte extracts as described ${ }^{(30)}$, using 
$20 \mu \mathrm{g}$ of protein and 3-8\% Tris-acetate gels (Invitrogen). Holocarboxylase-bound biotin in transblots was probed with fluorophore-labelled streptavidin; glyceraldehyde-3-phosphate dehydrogenase was used as a loading control $^{(4)}$. The membrane was scanned using the $800 \mathrm{CW}$ channel in an IR imaging system (Odyssey LI-COR) and band intensities were quantified by gel densitometry ${ }^{(30)}$.

Total RNA was extracted from lymphocytes using the ZR RNA MicroPrep (Zymo Research) following the manufacturer's instructions. RNA was reverse transcribed into complementary DNA using the High Capacity RNA-to-cDNA Kit (Applied Biosystems), following the manufacturer's instructions. The abundance of mRNA coding for the Na-dependent multivitamin transporter, monocarboxylate transporter 1 , holocarboxylase synthetase, ACC1 and MCC ( $\beta$ bchain) were quantified by quantitative real-time PCR using Quanta Perfecta Power SYBR Green and the cycle threshold method ${ }^{(4,34)}$. The abundance of GAPDH mRNA was used to normalise data for the efficiency of PCR. PCR primers were the same as reported previously $^{(30)}$. Our rationale for focusing on these five genes was that they play a role in biotin metabolism ${ }^{(1)}$ and because their expression depended on biotin in previous studies in human cell cultures ${ }^{(30)}$.

Concentrations of biotin in urine samples were quantified by the avidin-binding assay, as described ${ }^{(29)}$. As urine was collected as a spot urine sample (as opposed to 24-h collections), urinary biotin was normalised by the excretion of creatinine to account for possible incomplete collections and dilution effects. Creatinine was quantified using the standard picric acid method of Jaffe ${ }^{(35)}$. Concentrations of urinary organic acids were determined by a capillary electrophoresis method in our laboratory. Briefly, urine samples were thawed and centrifuged at $16100 \mathrm{~g}$ for $1 \mathrm{~min}$. A volume of $100 \mu \mathrm{l}$ of supernatant was lyophilised and weights of the freeze-dried samples were recorded for data normalisation. After being re-dissolved in $50 \mu \mathrm{l}$ of nanopure water, samples were injected into a $50 \mathrm{~cm}$ bare fused-silica capillary (inner diameter $50 \mu \mathrm{m}$ ) under the pressure of $0.5 \mathrm{psi}$ (pounds per square inch) for $5 \mathrm{~s}$. Various organic acids were resolved at $15 \mathrm{kV}$ and $25^{\circ} \mathrm{C}$ using a Beckman P/ACE MDQ capillary electrophoresis system (Beckman Coulter) equipped with a $200 \mathrm{~nm}$ UV filter using $250 \mathrm{~mm}$-phosphate buffer ( $\mathrm{pH}$ 6.0) with $0 \cdot 1$ mm-hexadecyltrimethylammonium bromide and $5 \%$ methanol (by vol.). Authentic standards of organic acid were purchased from Sigma-Aldrich. The following nine organic acids were quantified by a standard curve method and their urinary excretion was normalised by the excretion of creatinine: acetate, $\alpha$-ketoglutarate, butyrate, citrate, fumarate, malate, oxalate, succinate and 3-hydroxyisovaleric acid (3-HIA). Our rationale for focusing on these organic acids was that they are implicated in biotin-dependent pathways in intermediary metabolism and that, for some of them, links have been established with biotin deficiency $^{(1,36)}$.

\section{Statistical analysis}

To test for homogeneity of variances, Barlett's test or Levene's test was performed ${ }^{(37)}$. Data were $\log$ transformed if variances were heterogeneous; log transformation resulted in homogeneous variances. The significance of differences among groups was tested with one-way ANOVA and the Bonferroni method was used to adjust for multiple comparisons where appropriate $^{(37)}$. StatView 5.0.1 (SAS Institute) was used to perform all calculations. Differences are considered significant if $P<0 \cdot 05$. Data are expressed as mean and standard deviations, where SD represents sample standard deviation in all cases.

\section{Results}

\section{Holocarboxylases}

Biotinylated holo-MCC (molecular mass $=83 \mathrm{kDa}$ ) and holo-PCC (molecular mass $=80 \mathrm{kDa}$ ) from lymphocyte extracts were easily detectable in streptavidin blots and appear to be reliable markers for biotin status (Fig. 2(A)). Note that streptavidin blotting detects the biotinylated $\alpha$-chains of these carboxylases, but not the non-biotinylated $\beta$-chains. While the abundance of holo-pyruvate carboxylase also appeared to respond to dietary biotin, the signal produced by holo-pyruvate carboxylase was faint and hard to detect, suggesting that pyruvate carboxylase might not be a good marker for biotin status. Holo-ACC1 and ACC2 were not detectable in streptavidin blots of lymphocyte extracts, which is consistent with previous studies in human lymphoid cell cultures ${ }^{(29)}$. Gels were equally loaded, judged by the abundance of the housekeeping protein glyceraldehyde3-phosphate dehydrogenase.

Gel densitometry analysis of holo-MCC and holo-PCC is consistent with the notion that these proteins are good markers for biotin status. The abundance of holo-MCC (arbitrary units) was $4 \cdot 1$ (SD 2.6) in biotin-depleted subjects, 8.2 (SD 3.5) in biotin-sufficient subjects and 15.7 (SD 7.5) in biotin-supplemented subjects $(0.0003<P<0.0012$ with Bonferroni correction for all possible comparisons, $n 16$, Fig. 2(B)). Likewise, the abundance of holo-PCC (arbitrary units) was $4 \cdot 1$ (SD 1.9) in biotin-depleted subjects, 9.1 (SD 3.3) in biotin-sufficient subjects and $17 \cdot 0$ (SD 5.7) in biotin-supplemented subjects $(P<0.0003$ with Bonferroni correction for all possible comparisons, $n$ 16, Fig. 2(C)).

\section{Real-time $P C R$}

The abundance of mRNA coding for the biotin transporters, Na-dependent multivitamin transporter and monocarboxylate transporter 1; the biotin-dependent carboxylases, ACC1 and

Table 1. Urinary excretion of biotin in the three treatment groups $(n$ 16) (Mean values and standard deviations)

\begin{tabular}{llr}
\hline & \multicolumn{2}{c}{$\begin{array}{c}\text { Urinary biotin } \\
\text { (nmol biotin/ } \mu \text { mol creatinine) }\end{array}$} \\
\cline { 2 - 3 } Treatment & Mean & SD \\
\hline Biotin deficient & $0.52^{\mathrm{a}}$ & $1 \cdot 19$ \\
Biotin sufficient & $0 \cdot 74^{\mathrm{a}}$ & $1 \cdot 13$ \\
Biotin supplemented & $2 \cdot 54^{\mathrm{b}}$ & $2 \cdot 01$ \\
\hline
\end{tabular}

a,b Mean values with unlike superscript letters were significantly different $(0.0001<P<0.16)$. 
MCC ( $\beta$-chain); and holocarboxylase synthetase was not significantly different among the treatment groups (Fig. 3), contrary to previous observations in human cell cultures ${ }^{(30)}$. This apparent absence of effect can largely be explained by the large inter-individual variations among subjects. When men and women were analysed separately, the results were the same as for the pooled dataset (data not shown). We conclude that the abundance of mRNA coding for genes in biotin metabolism is not a good marker of biotin status in outpatient studies.

\section{Excretion of biotin in urine}

The urinary excretion of biotin was up to five times higher in biotin-supplemented subjects compared with the other treatment groups (Table 1). There was a trend towards higher urinary biotin in biotin-sufficient individuals compared with biotin-depleted individuals, but the difference was not statistically significant. This apparent absence of effect can largely be explained by the large inter-individual variations among subjects. When men and women were analysed separately, the results were the same as for the pooled dataset (data not shown). We conclude that the urinary excretion of biotin is a good marker for identifying supplement users, but is not a good marker to distinguish deficient from sufficient individuals in outpatient studies.

\section{Excretion of organic acids in urine}

The urinary excretion of 3-HIA was, on average, two times higher in biotin-deficient subjects compared with the other treatment groups (Fig. 4(A)). The use of 3-HIA as a marker of biotin status is limited by the following characteristics. First, the excretion of 3-HIA does not distinguish biotinsufficient and biotin-supplemented subjects. Second, for eight of the sixteen subjects, the urinary excretion of 3-HIA did not increase during biotin depletion compared with other phases (Fig. 4(B)), suggesting that the urinary excretion of 3-HIA has a tendency to produce false negatives in biotin-deficient subjects. For clarity, we show values only for those eight subjects for whom urinary 3-HIA did not respond to dietary biotin; for comparison, the figure also includes the data for one subject that showed the expected pattern of a higher excretion of 3-HIA during biotin depletion compared with other phases. When men and women were analysed separately, the results were the same as for the pooled dataset (data not shown).

Two of the remaining organic acids produced potentially interesting trends regarding their urinary patterns, although effects did not reach a statistically significant level. Urinary citrate was positively linked with biotin intake (Fig. 5(A)), while urinary malate was negatively linked with biotin intake (Fig. 5(B)), but none of these organic acids produced statistically significant effects due to large inter-individual variation. Results were the same when men and women were analysed separately. The urinary excretion of acetate, $\alpha$-ketoglutarate, butyrate, fumarate, oxalate and succinate did not depend on biotin intake.

\section{Dietary records}

The dietary intake of biotin was of the expected magnitude and well within the amount that can be bound by the avidin in the egg-white drinks. The intake of biotin over a 3-d period was 37.9 (SD 23.5) $\mu \mathrm{g}$ in biotin-depleted subjects, 65.2 (SD 71.4) $\mu \mathrm{g}$ in biotin-sufficient subjects and $49 \cdot 3$ (SD 29.2) $\mu \mathrm{g}$ in biotin-supplemented subjects ( $n$ 16), excluding the biotin that was added back to drinks. These values should be considered as crude estimates because there is currently no database that accurately quantifies biotin in foods ${ }^{(38,39)}$. Despite the limited accuracy of existing databases, the intake estimates reported here are consistent with the notion that subjects avoided foods rich in biotin throughout the study.

\section{Efficacy of washout}

Adjustment and washout periods were chosen to be sufficiently long to allow for biotin levels to return to baseline levels. When carboxylase-bound biotin in lymphocyte extracts
(A)

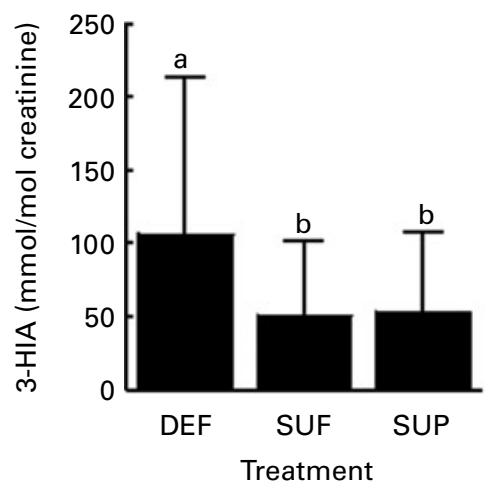

(B)

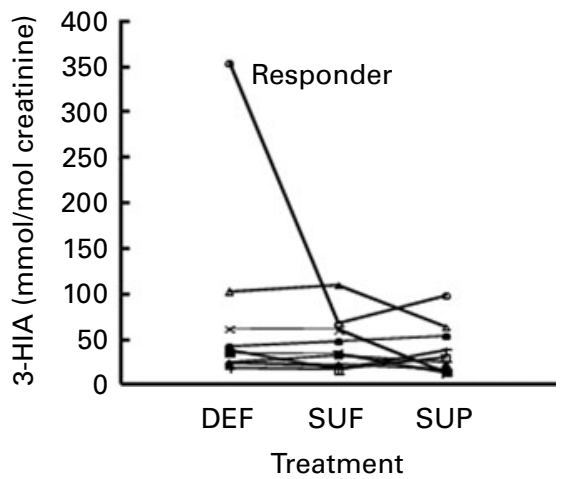

Fig. 4. (A) Average urinary excretion of 3-hydroxyisovaleric acid (3-HIA) in biotin-deficient (DEF), biotin-sufficient (SUF) and biotin-supplemented (SUP) healthy adults. Values are means, with standard deviations represented by vertical bars ( $n$ 16). ${ }^{a, b}$ Mean values with unlike letters were significantly different $(0.04<P<0.98)$. (B) Individual patterns of the eight subjects in whom urinary 3 -HIA did not respond to dietary biotin and in one of the subjects who exhibited the expected pattern of high urinary 3-HIA during biotin depletion (denoted 'Responder'). 

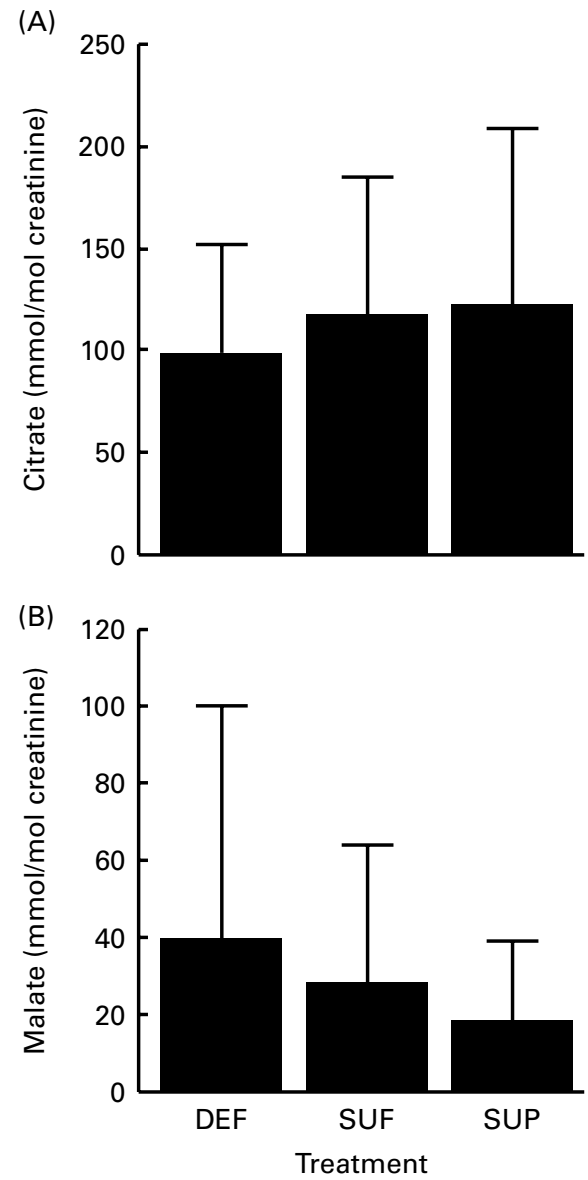

Fig. 5. Urinary excretion of $(A)$ citrate and (B) malate in biotin-deficient (DEF), biotin-sufficient (SUF) and biotin-supplemented (SUP) healthy adults. Values are means, with standard deviations represented by vertical bars $(0.4620<P<0.6943, n 16)$.

was probed with streptavidin, no apparent difference among the various phases was detected for any of the study subjects. Fig. 6 depicts the raw data from one subject as a representative example. The abundance of glyceraldehyde-3-phosphate dehydrogenase was used as a loading control. Likewise, the urinary excretion of biotin returned to baseline levels by the end of each washout period (approximately $1 \mathrm{nmol}$ biotin/ $\mu$ mol creatinine, assayed in randomly selected samples).

\section{Discussion}

The present paper is the first study that uses an outpatient feeding protocol in normal healthy adults to assess the effects of deficiency, sufficiency and supplementation of biotin on a comprehensive set of twenty variables from proteomics, transcriptomics and metabolomics. The study provides compelling evidence that the abundance of biotinylated (holo-)MCC and PCC in lymphocytes are the only markers that can reliably discriminate between states of moderate biotin deficiency and biotin sufficiency. This observation is consistent with previous studies in human lymphoid cell cultures, which also suggest that biotinylation of MCC and PCC is responsive to biotin intake $^{(29,30)}$. Greater than $50 \%$ of PCC in lymphocytes from biotin-depleted individuals is present in the biotin-free apoform, based on ex vitro cultures of lymphocytes from patients on biotin-free nutrition ${ }^{(40)}$. Previous studies in healthy adults $^{(41)}$ and rats ${ }^{(42)}$ also led to the conclusion that the activity of PCC is a good marker of biotin status in the population. Those studies used the incorporation of radiolabelled bicarbonate for assessing carboxylase activity, while in the present study we quantified the binding of biotin to carboxylases. In previous studies, we demonstrated that both carboxylase activity and carboxylase biotinylation correlate well ${ }^{(29)}$. However, we believe that the dependence on radiolabelled compounds is a meaningful limitation when using carboxylases as markers for biotin status. We also believe that streptavidin blots of carboxylases has the additional advantage of assessing multiple carboxylases in one single run, while activity assays require distinct analytical procedures for each of these enzymes ${ }^{(43)}$.

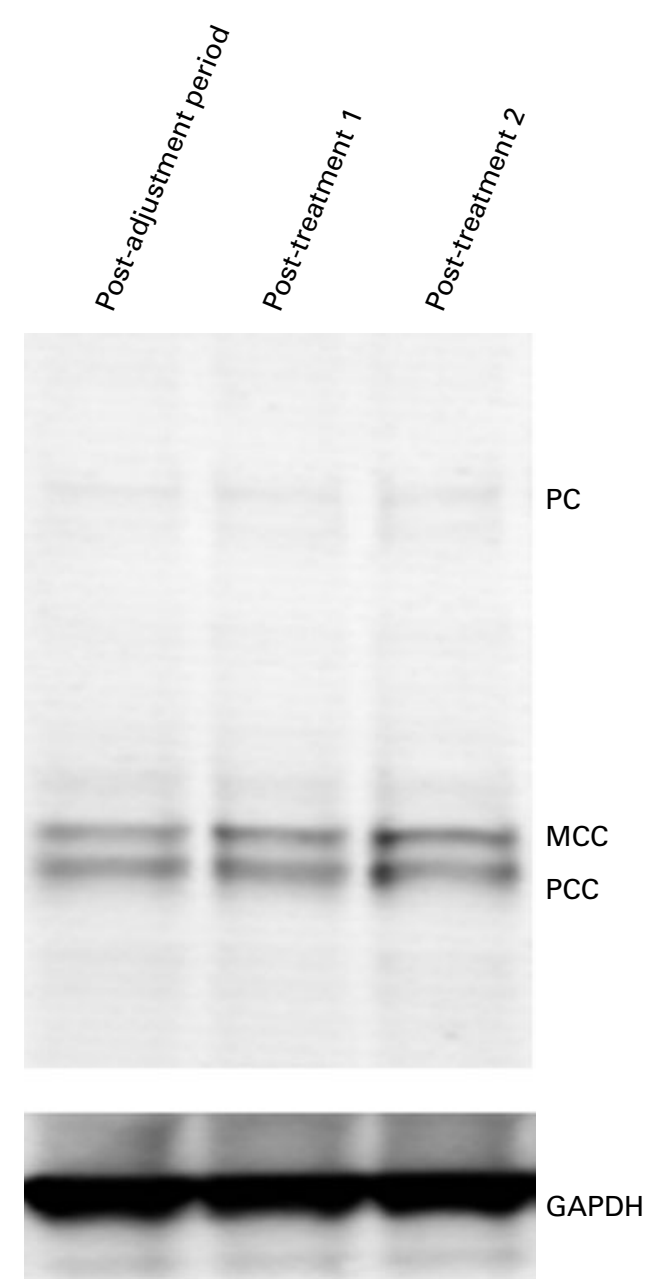

Fig. 6. Abundance of biotinylated (holo-)pyruvate carboxylase (PC), 3-methylcrotonyl-CoA carboxylase (MCC) and propionyl-CoA carboxylase (PCC) in lymphocytes from biotin-deficient, biotin-sufficient and biotinsupplemented subjects at the end of adjustment and washout phases (top gel). Glyceraldehyde-3-phosphate dehydrogenase (GAPDH) was used as the loading control (bottom gel). The image was cropped from an original full-size scanned picture. 
When biotin is insufficient during leucine metabolism, it shunts the pathway to an alternative pathway, leading to the formation of 3-HIA ${ }^{(1)}$. Urinary 3-HIA is generally considered a useful marker for biotin status ${ }^{(36,44,45)}$. Based on the findings in the present study, the usefulness of 3-HIA as a marker of biotin status needs to be re-evaluated because of the following concerns. First, urinary 3-HIA does not permit discriminating biotin-sufficient and biotin-supplemented individuals. Second, the average urinary excretion of 3-HIA was greater in biotin-deficient subjects compared with other treatment groups, but produced a meaningful number of false-negative results when looking at the individuals in the biotin-deficient group. More recently, carnitine conjugates of 3-HIA have been recommended as markers of biotin status ${ }^{(45-47)}$. Carnitine conjugates of 3-HIA were not tested in the present study, but might be a useful alternative to free 3-HIA. When validating carnitine conjugates in future studies, great care should be taken (a) to document inter-individual variation, (b) to test for false negatives in a population of biotin-depleted individuals and (c) to determine whether these conjugates discriminate between sufficient and supplemented individuals.

Some variables such as organic acids produced borderline significant changes of some markers in response to biotin intake. One could argue that these variables may produce statistically significant results if tested in a sample larger than sixteen subjects. Such concerns are formally correct. However, concerns would remain as to whether such variables are useful markers when trying to single out individuals with slightly lower and slightly higher biotin intake compared with the average intake in the sample.

One could consider using urinary biotin as a marker of biotin status. Our studies suggest that the urinary excretion of biotin reliably identifies biotin-supplemented subjects, but does not distinguish between biotin-depleted and biotinsufficient individuals. Also, urine contains biotin metabolites such as bisnorbiotin, biotin-D, L-sulphoxides, biotin sulphone, bisnorbiotin methyl ketone and tetranorbiotin-L-sulphoxide ${ }^{(48,49)}$. Typical biotin assays do not quantify these metabolites accurately, and their analysis is reserved for specialty laboratories.

Serum biotin concentration and biotinidase activity were not measured in the present study, based on the following lines of reasoning. Previous studies suggest that serum concentrations of biotin and biotin metabolites do not decrease in biotindeficient individuals ${ }^{(50)}$ and in patients on biotin-free total parenteral nutrition ${ }^{(40)}$ during reasonable periods of observation. Previous studies also suggest that the expression of biotinidase does not depend on biotin in human cell cultures $^{(30)}$.

Of the twenty potential markers of biotin status that were tested in the present study, quantification of mRNA abundance returned the least compelling results. None of the five genes from biotin turnover was linked in any way to biotin intake. This can be attributed to large inter-individual variations in the expression of these genes. This observation is in striking contrast to studies in cell cultures and laboratory animals, where the expression of these genes depended on biotin $^{(30,51,52)}$. We propose that the genetic diversity in human subjects and environmental factors create a unique scenario, and the studies in cell cultures and inbred animals need to be interpreted with care.

Collectively, we conclude that the number of reliable markers of biotin status is limited and that more groundwork needs to be done before human biotin requirements can be quantified with a reasonable level of certainty. No single marker is currently known that reliably distinguishes moderately biotin-deficient, biotin-sufficient and moderately biotinsupplemented individuals.

\section{Acknowledgements}

The present study was a contribution of the University of Nebraska Agricultural Research Division, supported in part by funds provided through the Hatch Act. Additional support was provided by National Institutes of Health grants (grant nos. DK063945, DK077816 and DK082476). The contributions of authors were as follows: J. Z., V. L. S., D. W. and D. G. designed the research; D. G. conducted the preliminary research; D. G. and W. K. E. conducted the research; B. H. L. conducted the capillary electrophoresis analyses; J. Z., D. W., D. G. and W. K. E. analysed the data; W. K. E. and J. Z. prepared the manuscript. All authors read, edited and approved the final manuscript. The authors declare that there is no conflict of interests.

\section{References}

1. Zempleni J, Wijeratne SS \& Hassan YI (2009) Biotin. Biofactors 35, 36-46.

2. Camporeale G, Shubert EE, Sarath G, et al. (2004) K8 and K12 are biotinylated in human histone H4. Eur J Biochem 271, 2257-2263.

3. Kobza K, Camporeale G, Rueckert B, et al. (2005) K4, K9, and $\mathrm{K} 18$ in human histone $\mathrm{H} 3$ are targets for biotinylation by biotinidase. FEBS J 272, 4249-4259.

4. Pestinger V, Wijeratne SSK, Rodriguez-Melendez R, et al. (2011) Novel histone biotinylation marks are enriched in repeat regions and participate in repression of transcriptionally competent genes. J Nutr Biochem 22, 328-333.

5. Rios-Avila L, Pestinger V \& Zempleni J (2012) K16-biotinylated histone $\mathrm{H} 4$ is overrepresented in repeat regions and participates in the repression of transcriptionally competent genes in human Jurkat lymphoid cells. J Nutr Biochem 23, 1559-1564.

6. Stanley JS, Griffin JB \& Zempleni J (2001) Biotinylation of histones in human cells: effects of cell proliferation. Eur J Biochem 268, 5424-5429.

7. Bailey LM, Ivanov RA, Wallace JC, et al. (2008) Artifactual detection of biotin on histones by streptavidin. Anal Biochem 373, 71-77.

8. Kuroishi T, Rios-Avila L, Pestinger V, et al. (2011) Biotinylation is a natural, albeit rare, modification of human histones. Mol Genet Metab 104, 537-545.

9. Camporeale G, Oommen AM, Griffin JB, et al. (2007) K12-biotinylated histone $\mathrm{H} 4$ marks heterochromatin in human lymphoblastoma cells. J Nutr Biochem 18, 760-768.

10. Filenko NA, Kolar C, West JT, et al. (2011) The role of histone $\mathrm{H} 4$ biotinylation in the structure and dynamics of nucleosomes. PLoS One 6, e16-299.

11. Bao B, Pestinger V, HY I, et al. (2011) Holocarboxylase synthetase is a chromatin protein and interacts directly 
with histone $\mathrm{H} 3$ to mediate biotinylation of $\mathrm{K} 9$ and $\mathrm{K} 18$. J Nutr Biochem 22, 470-475.

12. National Research Council (1998) Dietary Reference Intakes for Thiamin, Riboflavin, Niacin, Vitamin $B_{6}$, Folate, Vitamin $B_{12}$, Pantothenic Acid, Biotin, and Choline. Washington, DC: National Academy Press.

13. Zempleni J \& Mock DM (2000) Biotin. In Modern Analytical Methodologies on Fat and Water-Soluble Vitamins, pp. 389-409 [WO Song and GR Beecher, editors]. New York, NY: Wiley \& Sons, Inc.

14. Green NM (1975) Avidin. Adv Protein Chem 29, 85-133.

15. Mock N, Malik M, Stumbo P, et al. (1997) Increased urinary excretion of 3-hydroxyisovaleric acid and decreased urinary excretion of biotin are sensitive early indicators of decreased status in experimental biotin deficiency. Am J Clin Nutr 65, 951-958.

16. Sealey WM, Teague AM, Stratton SL, et al. (2004) Smoking accelerates biotin catabolism in women. Am J Clin Nutr 80, 932-935.

17. Weiner DL, Grier RE \& Wolf B (1985) A bioassay for determining biotinidase activity and for discriminating biocytin from biotin using holocarboxylase synthetase-deficient cultured fibroblasts. J Inherit Metab Dis 8, 101-102.

18. Wolf B, Heard GS, Weissbecker KA, et al. (1985) Biotinidase deficiency: initial clinical features and rapid diagnosis. Ann Neurol 18, 614-617.

19. Mock DM \& Dyken ME (1997) Biotin catabolism is accelerated in adults receiving long-term therapy with anticonvulsants. Neurology 49, 1444-1447.

20. Rathman SC, Eisenschenk S \& McMahon RJ (2002) The abundance and function of biotin-dependent enzymes are reduced in rats chronically administered carbamazepine. J Nutr 132, 3405-3410.

21. Mock DM \& Stadler DD (1997) Conflicting indicators of biotin status from a cross-sectional study of normal pregnancy. J Am Coll Nutr 16, 252-257.

22. Mock DM, Stadler D, Stratton S, et al. (1997) Biotin status assessed longitudinally in pregnant women. J Nutr $\mathbf{1 2 7}$ $710-716$.

23. Mock DM, Mock NI \& Dankle JA (1992) Secretory patterns of biotin in human milk. J Nutr 122, 546-552.

24. Camporeale G \& Zempleni J (2006) Biotin. In Present Knowledge in Nutrition, 9th ed., pp. 314-326 [BA Bowman and RM Russell, editors]. Washington, DC: International Life Sciences Institute.

25. Stratton SL, Henrich CL, Matthews NI, et al. (2012) Marginal biotin deficiency can be induced experimentally in humans using a cost-effective outpatient design. J Nutr 142, 22-26.

26. Baugh CM, Malone JH \& Butterworth CE Jr (1968) Human biotin deficiency: a case history of biotin deficiency induced by raw egg consumption in a cirrhotic patient. Am J Clin Nutr 21, 173-182.

27. Wolf B \& Heard GS (1991) Biotinidase deficiency. In Advances in Pediatrics, pp. 1-21 [L Barness and F Oski, editors]. Chicago, IL: Medical Book Publishers.

28. Zempleni J, Helm RM \& Mock DM (2001) In vivo biotin supplementation at a pharmacologic dose decreases proliferation rates of human peripheral blood mononuclear cells and cytokine release. J Nutr 131, 1479-1484.

29. Manthey KC, Griffin JB \& Zempleni J (2002) Biotin supply affects expression of biotin transporters, biotinylation of carboxylases, and metabolism of interleukin-2 in Jurkat cells. J Nutr 132, 887-892.

30. Kaur Mall G, Chew YC \& Zempleni J (2010) Biotin requirements are lower in human Jurkat lymphoid cells but homeostatic mechanisms are similar to those of HepG2 liver cells. J Nutr 140, 1086-1092.

31. Green NM (1965) A spectrophotometric assay for avidin and biotin based on binding of dyes by avidin. Biochem $J \mathbf{9 4}$, $23 \mathrm{c}-24 \mathrm{c}$

32. Durance TD (1991) Residual avidin activity in cooked egg white assayed with improved sensitivity. J Food Sci 56, 707-709.

33. Zempleni J \& Mock DM (1998) Uptake and metabolism of biotin by human peripheral blood mononuclear cells. Am J Physiol Cell Physiol 275, C382-C388.

34. Livak KJ \& Schmittgen TD (2001) Analysis of relative gene expression data using real-time quantitative PCR and the 2(-Delta Delta C(T)) method. Methods 25, 402-408.

35. O'Brien D, Ibbott FA \& Rodgerson DO (1968) Laboratory Manual of Pediatric Micro-biochemical Techniques, 4th ed. New York: Hoeber Medical Division, Harper \& Row, Publishers, Inc.

36. Mock DM, Henrich CL, Carnell N, et al. (2002) Indicators of marginal biotin deficiency and repletion in humans: validation of 3-hydroxyisovaleric acid excretion and a leucine challenge. Am J Clin Nutr 76, 1061-1068.

37. SAS Institute (1999) StatView Reference, 3rd ed. Cary, NC: SAS Publishing.

38. Staggs CG, Sealey WM, McCabe BJ, et al. (2004) Determination of the biotin content of select foods using accurate and sensitive HPLC/avidin binding. J Food Compost Anal 17, 767-776.

39. Food Standards Australia New Zealand (2010) NUTTAB 2010 Online Searchable Database. http://www.foodstandards.gov. $\mathrm{au} /$ consumerinformation/nuttab2010/nuttab2010onlinesearchabledatabase/onlineversion. $\mathrm{cfm}$ ?\&action $=$ search (accessed 25 June 2012).

40. Velazquez A, Zamudio S, Baez A, et al. (1990) Indicators of biotin status: a study of patients on prolonged total parenteral nutrition. Eur J Clin Nutr 44, 11-16.

41. Mock DM, Henrich CL, Carnell N, et al. (2002) Lymphocyte propionyl-CoA carboxylase and accumulation of odd-chain fatty acid in plasma and erythrocytes are useful indicators of marginal biotin deficiency. $J$ Nutr Biochem 13, 462-470.

42. Mock DM \& Mock NI (2002) Lymphocyte propionyl-CoA carboxylase is an early and sensitive indicator of biotin deficiency in rats, but urinary excretion of 3-hydroxypropionic acid is not. J Nutr 132, 1945-1950.

43. Zempleni J, Trusty TA \& Mock DM (1997) Lipoic acid reduces the activities of biotin-dependent carboxylases in rat liver. J Nutr 127, 1776-1781

44. Mock NI, Mock DM, Malik M, et al. (1995) Urinary excretion of biotin and 3-hydroxyisovaleric acid (3-HIA) are early indicators of biotin deficiency. FASEB J 9, A985 [abstract].

45. Stratton SL, Horvath TD, Bogusiewicz A, et al. (2011) Urinary excretion of 3-hydroxyisovaleryl carnitine is an early and sensitive indicator of marginal biotin deficiency in humans. J Nutr 141, 353-358.

46. Horvath TD, Stratton SL, Bogusiewicz A, et al. (2010) Quantitative measurement of urinary excretion of 3-hydroxyisovaleryl carnitine by LC-MS/MS as an indicator of biotin status in humans. Anal Chem 82, 9543-9548.

47. Mock DM, Stratton SL, Horvath TD, et al. (2011) Urinary excretion of 3-hydroxyisovaleric acid and 3-hydroxyisovaleryl carnitine increases in response to a leucine challenge in marginally biotin-deficient humans. J Nutr 141, $1925-1930$. 
48. Mock DM, Lankford GL \& Cazin J Jr (1993) Biotin and biotin analogs in human urine: biotin accounts for only half of the total. J Nutr 123, 1844-1851.

49. Zempleni J, McCormick DB \& Mock DM (1997) Identification of biotin sulfone, bisnorbiotin methyl ketone, and tetranorbiotinL-sulfoxide in human urine. Am J Clin Nutr 65, 508-511.

50. Mock DM \& Mock NI (1997) Serum concentrations of bisnorbiotin and biotin sulfoxide increase during both acute and chronic biotin supplementation. J Lab Clin Med 129, 384-388.
51. Rodriguez-Melendez R, Cano S, Mendez ST, et al. (2001) Biotin regulates the genetic expression of holocarboxylase synthetase and mitochondrial carboxylases in rats. $J$ Nutr 131, 1909-1913.

52. Solorzano-Vargas RS, Pacheco-Alvarez D \& Leon-Del-Rio A (2002) Holocarboxylase synthetase is an obligate participant in biotin-mediated regulation of its own expression and of biotin-dependent carboxylases mRNA levels in human cells. Proc Natl Acad Sci U S A 99, 5325-5330. 